\title{
Immunohistochemical detection of XIAP and $p 63$ in adenomatous hyperplasia, atypical adenomatous hyperplasia, bronchioloalveolar carcinoma and well-differentiated adenocarcinoma
}

\author{
Maoxin $\mathrm{Wu}^{1}$, Lurmag $\operatorname{Orta}^{1}$, Joan $\mathrm{Gil}^{1}$, Gan $\mathrm{Li}^{1}$, Alice $\mathrm{Hu}^{1}$ and David E Burstein ${ }^{1,2}$ \\ ${ }^{1}$ Department of Pathology, Mount Sinai School of Medicine, New York, NY, USA and ${ }^{2}$ Department \\ of Oncologic Sciences, Mount Sinai School of Medicine, New York, NY, USA
}

\begin{abstract}
The critical distinction of bronchioloalveolar carcinoma (BAC), well-differentiated adenocarcinoma (WDAC) of lung, adenomatous hyperplasia (AH) and atypical adenomatous hyperplasia (AAH), is based on morphological criteria alone, and is therefore potentially subjective. We examined expression of two markers, X-linked inhibitor of apoptosis protein (XIAP), the most potent of the inhibitor of apoptosis protein (IAP) family, and p63, a marker of bronchial reserve cells (BRC) and squamous cells, in these entities. H\&E slides of 37 tissue blocks from 27 patients were reviewed and classified as AH $(n=7)$, AAH $(n=8)$, BAC $(n=9)$ and WDAC $(n=13)$. Immunostaining was performed on $4 \mu \mathrm{m}$ sections with monoclonal anti-XIAP and monoclonal anti-p63. Granular or heterogeneous cytoplasmic staining for XIAP and nuclear staining for p63 were considered positive. Neither XIAP nor p63 were detected in normal lung alveolar cells. All seven AHs were negative for XIAP and negative or focally positive for p63. All eight AAHs were positive for XIAP and displayed p63 positivity in scattered cells. All BACs displayed XIAP positivity, which ranged from focal/weak to diffuse/strong. p63 was negative in seven and focally positive in two of nine BACs. Twelve of 13 WDACs showed XIAP positivity in a similar pattern to BAC; all were negative for p63. One aberrant case diagnosed on $H$ \& $E$ as WDAC was negative for XIAP but strongly positive for p63. Significant XIAP expression appears to be useful for distinguishing AAH from AH. Commonality of XIAP staining in AAH, BAC and WDAC supports the possibility that AAH may be a premalignant lesion. The rarity of p63 expression confirms previous reports and supports a nonbronchial histogenesis of these entities. In contrast, diffuse p63 staining may facilitate the identification of rare cases that may have been misclassified as alveolar in origin based on morphology but may be of BRC origin.
\end{abstract}

Modern Pathology (2008) 21, 553-558; doi:10.1038/modpathol.2008.5; published online 1 February 2008

Keywords: XIAP; p63; adenomatous hyperplasia; atypical adenomatous hyperplasia; bronchioloalveolar carcinoma and well-differentiated adenocarcinoma

The distinction of bronchioloalveolar carcinoma (BAC), well-differentiated adenocarcinoma (WDAC) of lung, atypical adenomatous hyperplasia (AAH) and adenomatous hyperplasia $(\mathrm{AH})$, is critical for patient management, but can be difficult based upon histopathological features alone. AAH in particular is problematic in several aspects: atypia is a

Correspondence: Dr M Wu, MD, PhD, Department of Pathology, Mount Sinai School of Medicine, One Gustave L. Levy Place, New York, NY 10029-6574, USA.

E-mail: maoxin.wu@mssm.edu

Received 30 August 2007; revised 20 December 2007; accepted 31 December 2007; published online 1 February 2008 subjective term-cells that are atypical to some may be considered by others to be within normal limits. AAH is defined in the 2001 WHO classification $^{1}$ as a lesion that is less than $5 \mathrm{~mm}$, with mildly to moderately atypical cells lining alveoli and thickening of alveolar walls in the absence of underlying interstitial disease; however, these criteria have not been applied uniformly by all practitioners. Second, no objective markers have been described that may facilitate the distinction between reactive atypia and true pre-malignant atypia, or the distinction of AH from AAH. Whether the atypia in $\mathrm{AAH}$ represents true pre-malignant change is also unresolved. Another issue is the 
distinction between AAH and BAC. Based on the WHO 2001 classification, the only distinction between $\mathrm{AAH}$ and BAC is the size of the lesion, ie less than or greater than $5 \mathrm{~mm}$ in diameter, respectively. We report herein an examination of two markers, p63 and X-linked inhibitor of apoptosis protein (XIAP) in this spectrum of lung lesions.

Eight human inhibitor of apoptosis proteins (IAPs) have been discovered, of which XIAP is the most potent. ${ }^{2-4}$ The first described IAP, encoded by a baculovirus gene, was shown to protect virally infected cells and enhance viral replication. ${ }^{2}$ XIAP blocks activation of apoptosis-promoting caspases 3, 8 and 9, thereby blocking both the intrinsic and extrinsic apoptotic pathways. ${ }^{3,5}$ XIAP is commonly overexpressed in cancer cell lines; overexpression confers resistance to apoptosis-inducing chemotherapeutic agents and radiation. XIAP-inhibitory siRNA, antisense constructs or small molecules restore chemosensitivity in a variety of XIAP-overexpressing cell lines and in some cases induce apoptosis of tumor cells. ${ }^{6-11}$ XIAP expression correlates with clinical aggressiveness in acute myelogenous leukemia, diffuse large B-cell lymphoma and renal cell carcinoma. ${ }^{12-14}$ XIAP is expressed in some normal tissues; ${ }^{15}$ however, XIAP-knockout mice lack any significant pathology, suggesting normal cells function properly in its absence. For all of the above reasons, XIAP is considered an attractive target for drug discovery. XIAP reagents have been identified and are in preclinical as well as clinical trial testing. ${ }^{4,5,16,17}$

XIAP expression in non-small cell lung carcinoma has been reported in cell culture and animal models. ${ }^{18-21}$ However, immunohistochemical studies specifically addressing XIAP expression in human lung adenocarcinoma and alveolar hyperplasias are lacking. An immunocytochemical study of body cavity effusions and washes performed in our laboratory detected XIAP in eight out of nine metastatic non-small cell carcinomas of pulmonary origin, ${ }^{22}$ raising the possibility that XIAP may play a role in the pathogenesis or progression of lung adenocarcinoma.

p63 is a p53-homologous gene implicated in maintaining stem and basal/undifferentiated cell populations in squamous and other epithelia. In normal lung, p63 is strongly expressed in bronchial reserve cells, squamous metaplasia, squamous cell carcinoma and adenosquamous carcinoma; in $\mathrm{AAH}$ and lung adenocarcinoma, expression of p63 is less frequent and present in scattered cells, ${ }^{23-28}$ with most studies reporting positivity in fewer than $20 \%$ of adenocarcinomas.

We describe below patterns of immunohistochemical detectability of XIAP and p63 in AH, AAH, BAC and WDAC that may have implications for pathogenesis and may facilitate differential diagnosis in difficult cases, as well as shed some light on new options for therapy and clinical management of these lesions.

\section{Materials and methods}

Hematoxylin and eosin stained slides of 37 formalinfixed paraffin-embedded tissue blocks from 27 lung wedge excisions and lobectomies were reviewed by three anatomic pathologists, including an experienced pulmonary pathologist (JG), and were classified as $\mathrm{AH}(n=7)$, AAH $(n=8)$, BAC $(n=9)$ and WDAC $(n=13)$. Ten patients had multiple lesions ranging from $\mathrm{AH}$ to WDAC. Four of $10(40 \%)$ patients had both $\mathrm{AH}$ and adenocarcinoma and 2 of 10 patients had AAH associated with carcinomas.

In all sections, adjacent normal lung served as internal control for p63 (strong staining in bronchial reserve cells ${ }^{27}$ ), and a colon adenocarcinoma was a positive control for XIAP. Immunostaining was performed by standard citrate-based antigen retrieval followed by incubation with either monoclonal antiXIAP (clone 48; BD Biosciences, San Jose, CA, USA) diluted 1:125 in PBS plus $0.2 \%$ bovine serum albumin plus $5 \%$ goat serum, at $4^{\circ} \mathrm{C}$ for $72 \mathrm{~h}$ or monoclonal anti-p63 (4A4; Santa Cruz, Santa Cruz, CA, USA) diluted 1:4000 in PBS with $0.1 \%$ bovine serum albumin and 5\% non-fat dry milk overnight at room temperature, followed by incubation with EnVision-Plus reagents (Dako, Carpinteria, CA, USA) and diaminobenzidine as chromagen; granular or nonhomogeneous cytoplasmic staining for XIAP and nuclear staining for p63 were considered positive. The extensiveness of both XIAP and p63 staining was recorded as follows: negative; $1+$ : $<10 \%$ positivity; $2+$ : $10-50 \%$ positivity and $3+$ : $51-100 \%$ positivity. XIAP staining intensity was graded as negative; weak $(1+)$; moderate $(2+)$ or strong $(3+)$ positivity. For p63, nuclear staining was usually strong, therefore was graded as negative or positive.

\section{Results}

In normal lung, XIAP was consistently non-staining in normal alveolar epithelium (Figure 1a). In some cases, XIAP positivity was found in the apical cytoplasm of ciliated bronchial cells (Figure 1b), consistent with reports by others. ${ }^{15}$ p63 did not stain normal alveolar epithelium (Figure 1c), but strongly stained subsurface reserve cells in bronchi and bronchioles (Figure 1d).

All seven AHs were XIAP negative (Figure 2c) and displayed either p63 negativity (Figure 2d) or focal positivity.

Eight of eight AAHs (100\%) were XIAP-positive with positivity ranging from $1+$ to $3+$ in extent and intensity (Figure 3c: $3+$ ). Eight of eight AAHs $(100 \%)$ displayed nuclear p63 positivity in rare scattered atypical cells (Figure 3d) and five out of eight cases also showed weak cytoplasmic staining with anti-p63, which was considered nonspecific (see Discussion).

Nine of nine BACs (100\%) displayed XIAP positivity that ranged from focal/weak to diffuse/ 
strong (Figure 4c, 3+); p63 was negative (Figure 4d) in seven of nine BACs $(78 \%)$ and focally positive in two of nine $(22 \%)$. All 12 WDACs displayed XIAP staining in a similar pattern to BAC (Figure 5c, $3+$ ); all were negative for p63 (Figure 5d).

In some cases with both AAH and carcinoma present, expression of XIAP was stronger in AAH immediately adjacent to the carcinoma compared with the nonadjacent regions.

One lesion studied did not appear to conform to the aforementioned staining patterns. It was a $6 \mathrm{~mm}$ lesion originally classified as AAH and reclassified as WDAC in the course of this study using histopathological criteria. On H\&E stain, this lesion consisted of small gland-like nests of medium-sized pleomorphic atypical cells in a fibrous background, and, notably, appeared not to have lepidic pattern of spread characteristic of AH, AAH, BAC and WDAC. Unlike other WDACs or AAHs, it was XIAP-negative (Figure 6c) and was strongly and diffusely positive for p63 (Figure 6d). The possible significance of case is discussed below.
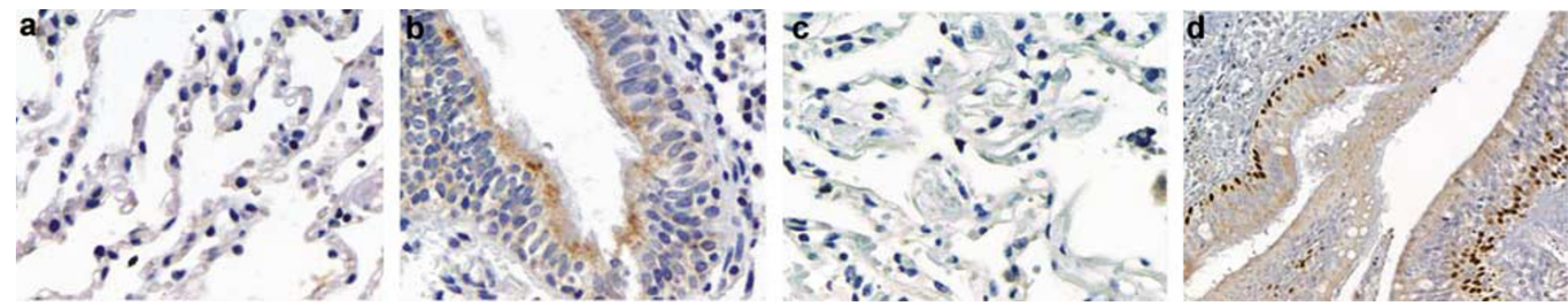

Figure 1 (a) Normal lung parenchyma with negative XIAP staining. (b) Normal bronchial epithelium with positive XIAP staining in apical pattern. (c) Normal lung parenchyma with virtually negative p63 staining. (d) Normal bronchial epithelium with positive p63 staining in the basal reserve cells.
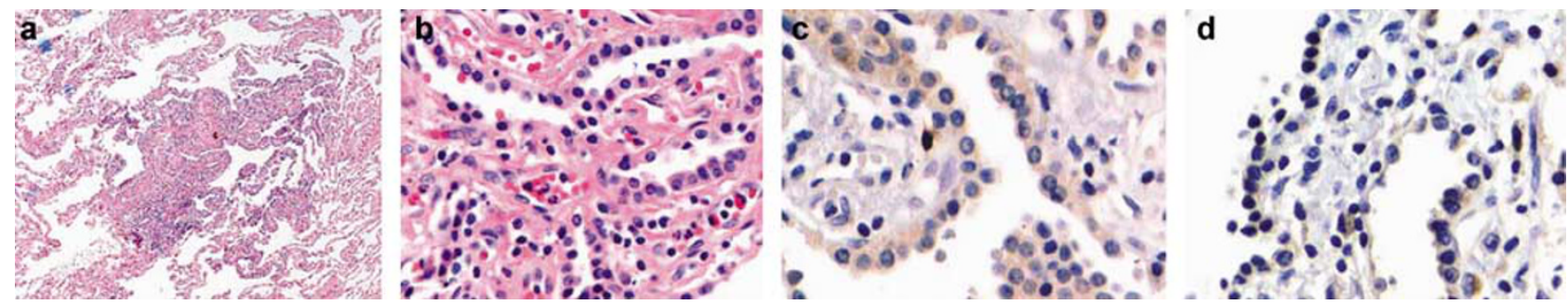

Figure 2 (a) Low-power view of AH with H\&E staining. (b) High-power view of AH with H\&E staining. (c) AH with virtually negative XIAP staining. (d) AH with virtually negative p63 staining.
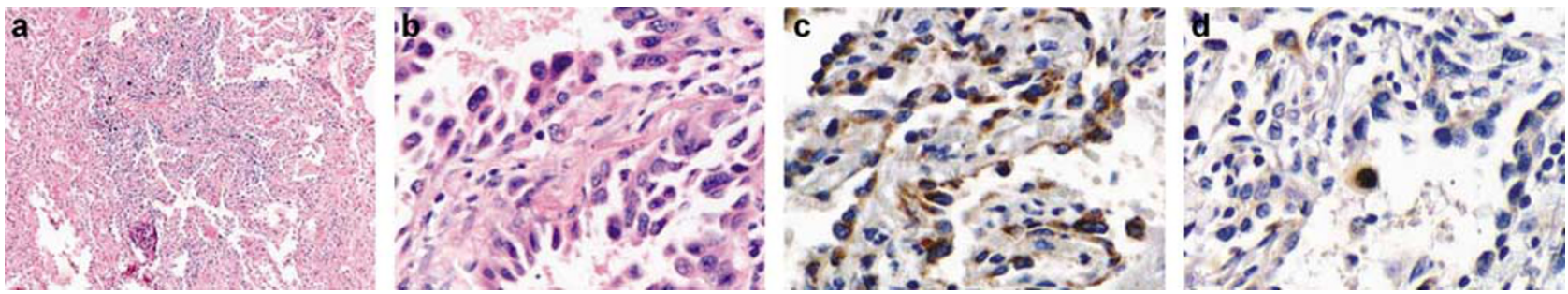

Figure 3 (a) Low-power view of AAH with H\&E staining. (b) High-power view of AAH with H\&E staining. (c) AAH with positive XIAP staining in the atypical cells. (d) AAH with occasional p63-positive nucleus.
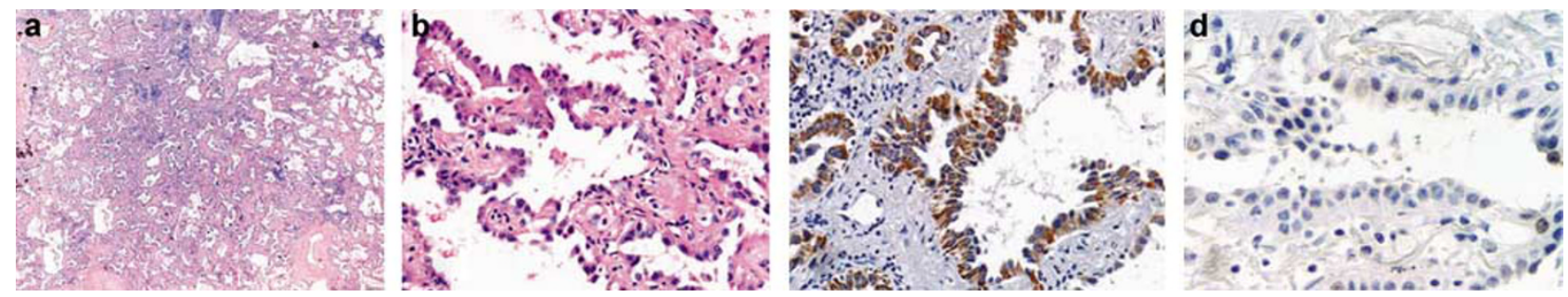

Figure 4 (a) Low-power view of BAC with H\&E staining. (b) High-power view of BAC with H\&E staining. (c) BAC with positive XIAP staining. (d) BAC with negative p63 staining. 

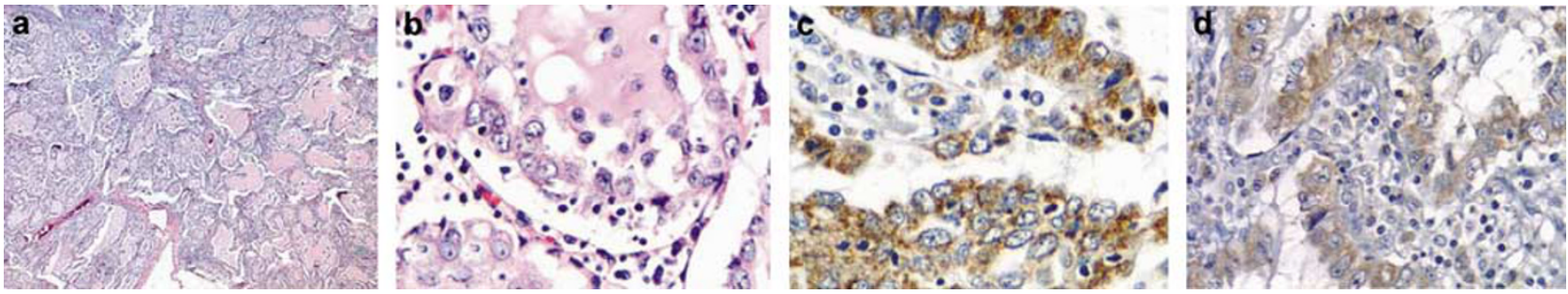

Figure 5 (a) Low-power view of WDAC with H\&E staining. (b) High-power view of WDAC with H\&E staining. (c) WDAC with positive XIAP. (d) WDAC with negative p63.
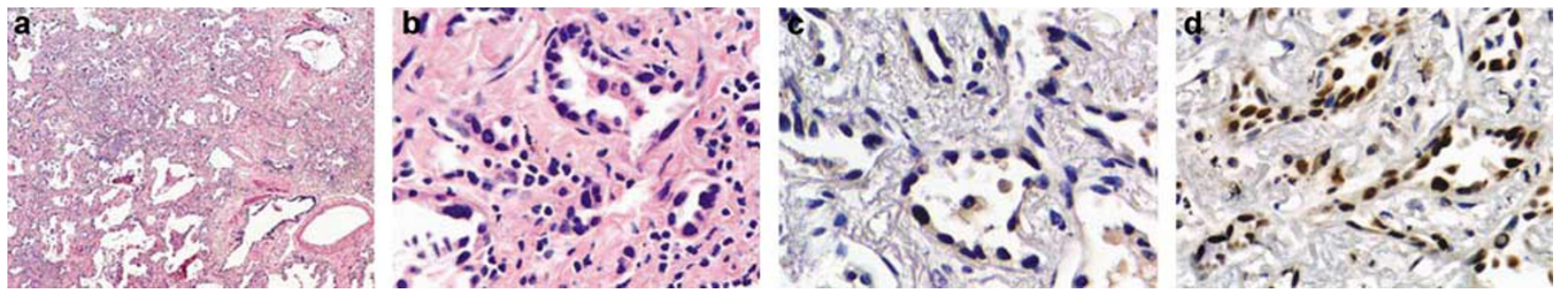

Figure 6 (a) H\&E stained low-power view of the controversial case; (b) H\&E stained high-power view of the case; (c) negative XIAP staining; (d) positive p63 staining.

\section{Discussion}

Expression of XIAP in AAH, BAC and WDAC but not in $\mathrm{AH}$ and normal lung alveolar tissue raises the possibility that AAH could represent a premalignant precursor to pulmonary adenocarcinoma. Although the number of cases in this study is small, XIAP expression appears to be of potential clinical use as a marker for distinguishing AH from AAH. Positive staining of XIAP in $100 \%$ of AAH compared with negative staining in $100 \%$ of AH suggests that the nature of cells in 'atypical adenomatous hyperplasia' may not be simply reactive and hyperplastic. Although the suggestion has been made that AAH may be a premalignant lesion based on chromosomal abnormalities, loss of LKB1 expression, overexpression of cyclin D1 and decreased p16 expression, ${ }^{29,30}$ proof has been lacking. Given the antiapoptotic function of XIAP, the present study links AAH, but not $\mathrm{AH}$ to defective apoptotic pathways that are a frequent and well-described aspect of malignancy.

The present findings also disclose a correlation of XIAP expression with atypical cytomorphology among these lesions: in $\mathrm{AH}$, in which the cells are benign appearing with small and uniform nuclei, XIAP is negative. In contrast, the cells in AAH are atypical with nuclear enlargement, pleomorphism and irregular nuclear contour and are positive for XIAP. The correlation of atypia with XIAP positivity may justify alternate proposed terminology for AAH, namely alveolar cell dysplasia or alveolar intraepithelial neoplasia. Indeed, the commonality of XIAP positivity blurs the distinction of $\mathrm{AAH}$ and BAC; other molecular findings may be required to determine whether one or both entities are indeed malignant, but differ only in the size of the lesion. In other studies, we have found immunohistochemically detectable XIAP in subsets of preneoplastic lesions of breast, head and neck, and uterine cervix. ${ }^{31-33}$

The reported prevalence of p63 positivity in lung adenocarcinoma varies considerably among published studies, but most suggest less than 20\% prevalence and moreover positivity is found in scattered cells rather than diffuse. In the present study, p63-positive cells were found to be scattered in $\mathrm{AH}$ and in $\mathrm{AAH}$ but rare in BAC and negative in WDAC (with the exception of the case discussed below). Although p63 is a transcription factor, for which nuclear staining alone was considered to be specific, we noted p63 cytoplasmic staining of undetermined relevance or significance in 5 of 8 AAH, 7 of 9 BAC and 3 of 12 WDAC (data not shown). Of note, one report suggests that cytoplasmic p63 staining correlates with worse prognosis in lung adenocarcinoma. ${ }^{34}$

As p63 is consistently positive in bronchial reserve cells and squamous carcinomas, ${ }^{24-28}$ strong diffuse positivity of p63 combined with XIAP negativity in one case interpreted morphologically to be WDAC was of interest, as this phenotype was essentially the reverse of that of other WDACs. Unlike other reported p63-positive lung adenocarcinomas, in which p63 positivity is present only in scattered cells, ${ }^{27,28}$ the present lesion was p63positive in virtually all tumor cells (Figure 6d). Of interest, a similar-appearing lesion described by Sheikh et $a l^{23}$ expressed p63 in $80 \%$ of cell nuclei, and was also a case in which $\mathrm{AH}$ with atypia was revised to a diagnosis of adenocarcinoma. That case and the present case raise the question of whether or 
Table 1 Summary of XIAP and p63 immuno-expression in lung adenocarcinoma-related lesions

\begin{tabular}{|c|c|c|}
\hline & $X I A P$ & p63 \\
\hline \multirow[t]{2}{*}{ Normal lung tissue $(n=37)$} & Negative in normal alveolar parenchyma & Negative in normal alveolar parenchyma \\
\hline & Positive in columnar bronchial cells (sporadic/apical) & Positive in bronchial reserve cells \\
\hline $\mathrm{AH}(n=7)$ & $100 \%(7 / 7):$ negative & Negative or rarely positive \\
\hline $\mathrm{AAH}(n=8)$ & $100 \%(8 / 8): 1+$ to $3+$ extent or intensity & $100 \%(8 / 8)$ positive in scattered cells \\
\hline $\operatorname{BAC}(n=9)$ & $100 \%(9 / 9): 1+$ to $3+$ extent or intensity & $\begin{array}{l}78 \%(7 / 9) \text { negative } \\
22 \%(2 / 9) \text { positive in scattered cells }\end{array}$ \\
\hline WDAC $(n=12)$ & $100 \%(12 / 12): 2+$ to $3+$ & $100 \%(12 / 12):$ negative \\
\hline Controversial case $(n=1)$ & Negative & Diffusely positive \\
\hline
\end{tabular}

not the p63-positive malignant-appearing cells are of bronchial reserve cell rather than alveolar origin. Whether such lesions constitute a previously unrecognized entity, and whether these are nonneoplastic, pre-malignant or malignant cannot be ascertained at present (Table 1).

\section{Acknowledgement}

This work is supported by a generous bequest of the Estate of Hilda Leven (DEB).

\section{References}

1 Brambilla E, Travis WD, Colby TV, et al. The new world health organization classification of lung tumours. Eur Respir J 2001;18:1059-1068.

2 Crook NE, Clem RJ, Miller LK. An apoptosis-inhibiting baculovirus gene with a zinc finger-like motif. J Virol 1993;67:2168-2174.

3 Yang YL, Li XM. The IAP family: endogenous caspase inhibitors with multiple biological activities. Cell Res 2000;10:169-177.

4 Holcik M, Gibson H, Korneluk RG. XIAP: apoptotic brake and promising therapeutic target. Apoptosis 2001;6:253-261.

5 Schimmer AD, Dalili S, Batey RA, et al. Targeting XIAP for the treatment of malignancy. Cell Death Differ 2006;13:179-188.

6 Berezovskaya O, Schimmer AD, Glinskii AB, et al. Increased expression of apoptosis inhibitor protein XIAP contributes to anoikis resistance of circulating human prostate cancer metastasis precursor cells. Cancer Res 2005;65:2378-2386.

7 Holcik M, Yeh C, Korneluk RG, et al. Translational upregulation of X-linked inhibitor of apoptosis (XIAP) increases resistance to radiation induced cell death. Oncogene 2000;19:4174-4177.

8 Tong QS, Zheng LD, Wang L, et al. Downregulation of XIAP expression induces apoptosis and enhances chemotherapeutic sensitivity in human gastric cancer cells. Cancer Gene Ther 2005;12:509-514.

9 Chawla-Sarkar M, Bae SI, Reu FJ, et al. Down regulation of Bcl-2, FLIP or IAPs (XIAP and survivin) by siRNAs sensitizes resistant melanoma cells to Apo2L/TRAIL-induced apoptosis. Cell Death Differ 2004;11:915-923.

10 Sasaki H, Sheng Y, Kotsuji F, et al. Down-regulation of $\mathrm{X}$-linked inhibitor of apoptosis protein induces apop- tosis in chemoresistant human ovarian cancer cells. Cancer Res 2000;60:5659-5666.

11 McManus DC, Lefebvre CA, Cherton-Horvat G, et al. Loss of XIAP protein expression by RNAi and antisense approaches sensitizes cancer cells to functionally diverse chemotherapeutics. Oncogene 2004;23: 8105-8117.

12 Kim JS, Eom JI, Cheong JW, et al. Protein kinase CK2alpha as an unfavorable prognostic marker and novel therapeutic target in acute myeloid leukemia. Clin Cancer Res 2007;13:1019-1028.

13 Muris JJ, Cillessen SA, Vos W, et al. Immunohistochemical profiling of caspase signaling pathways predicts clinical response to chemotherapy in primary nodal diffuse large B-cell lymphomas. Blood 2005;105:2916-2923.

14 Mizutani Y, Nakanishi H, Li YN, et al. Overexpression of XIAP expression in renal cell carcinoma predicts a worse prognosis. Int J Oncol 2007;4:919-925.

15 Vischioni B, van der Valk P, Span SW, et al. Expression and localization of inhibitor of apoptosis proteins in normal human tissues. Hum Pathol 2006; 37:78-86.

16 Kamsteeg M, Rutherford T, Sapi E, et al. Phenoxodiol-an isoflavone analog-induces apoptosis in chemoresistant ovarian cancer cells. Oncogene 2003; 22:2611-2620.

17 Rutherford TD, Makkenchery A, Mor G. Phenoxodiol phase Ib/II study in patients with recurrent ovarian cancer that are resistant to second line chemotherapy. J Soc Gynecol Invest 2004;11:254.

18 Checinska A, Hoogeland BS, Rodriguez JA, et al. Role of XIAP in inhibiting cisplatin-induced caspase activation in non-small cell lung cancer cells: a small molecule Smac mimic sensitizes for chemotherapyinduced apoptosis by enhancing caspase-3 activation. Exp Cell Res 2006;313:1215-1224.

19 Dong F, Guo W, Zhang L, et al. Downregulation of XIAP and induction of apoptosis by the synthetic cyclin-dependent kinase inhibitor GW8510 in nonsmall cell lung cancer cells. Cancer Biol Ther 2006;5: 165-170.

20 Cao C, Mu Y, Hallahan DE, et al. XIAP and survivin as therapeutic targets for radiation sensitization in preclinical models of lung cancer. Oncogene 2004;23: 7047-7052.

$21 \mathrm{Hu}$ Y, Cherton-Horvat G, Dragowska V, et al. Antisense oligonucleotides targeting XIAP induce apoptosis and enhance chemotherapeutic activity against human lung cancer cells in vitro and in vivo. Clin Cancer Res 2003;9:2826-2836.

$22 \mathrm{Wu}$ M, Yuan S, Szporn AH, et al. Immunocytochemical detection of XIAP in body effusion and washes. Mod Pathol 2005;18:1618-1622. 
23 Sheikh HA, Fuhrer K, Cieply K, et al. P63 expression in assessment of bronchioloalveolar proliferations of the lung. Mod Pathol 2004;17:1134-1140.

$24 \mathrm{Wu}$ M, Wang B, Gil J, et al. p63 and TTF-1 Immunostaining: a useful marker panel for distinguishing small cell carcinoma of lung from poorly differentiated squamous cell carcinoma of lung. Am J Clin Pathol 2003;119:696-702.

25 Shtilbans V, Szporn AH, Wu M, et al. p63 immunostaining in destained bronchoscopic cytologic specimen. Diagn Cytopathol 2005;32:198-203.

$26 \mathrm{Wu} \mathrm{M}$, Zhang D, Szporn AH, et al. Cytology applications of p63 and TTF-1 immunostaining in differential diagnosis of poorly differentiated primary and secondary lung cancers. Diagn Cytopathol 2005; 33:1-5.

27 Wang BY, Gil J, Kaufman D, et al. P63 in pulmonary epithelium, pulmonary squamous neoplasms, and other pulmonary tumors. Hum Pathol 2002;33:921-926.

28 Pelosi G, Pasini F, Stenholm CO. p63 immunoreactivity in lung cancer: yet another player in the development of squamous cell carcinomas? J Pathol 2002;198:100-108.
29 Ghaffar H, Sahin F, Sanchez-Cepedes M, et al. LKB1 protein expression in the evolution of glandular neoplasia of the lung. Clin Cancer Res 2003;9: 2998-3003.

30 Tominaga M, Sueoka N, Irie K, et al. Detection and discrimination of preneoplastic and early stages of lung adenocarcinoma using hnRNP B1 combined with the cell cycle-related markers p16, cyclin D1, and Ki67. Lung Cancer 2003;40:45-53.

31 Jaffer S, Orta L, Sunkara S, et al. Immunohistochemical detection of anti-apoptotic protein XIAP in mammary carcinoma. Hum Pathol 2007;38:864-870.

32 Nagi C, Xiao G-Q, Li G, et al. Immunohistochemical detection of XIAP in head and neck squamous cell carcinoma. Annal Diagn Pathol 2007;11:402-406.

33 Burstein DE, Idrees MT, Li G, et al. Immunohistochemical detection of XIAP in cervical squamous intraepithelial neoplasia and squamous carcinoma. Annal Diagn Pathol (in press).

34 Narahashi T, Niki T, Wang T, et al. Cytoplasmic localization of p63 is associated with poor patient survival in lung adenocarcinoma. Histopathology 2006;49:349-357. 